\title{
Polarization Diffraction Grating Produced by Femtosecond Laser Nanostructuring in Glass
}

\author{
Martynas Beresna and Peter G. Kazansky \\ Optoelectronics Research Centre, University of Southampton, SO17 1BJ, United Kingdom \\ pgk@orc.soton.ac.uk
}

\begin{abstract}
We demonstrate polarization sensitive diffractive optical element fabrication by femtosecond direct writing in the bulk of silica glass. Modulation of the anisotropic properties is produced by controlling light-induced self-assembled nano-gratings.

(C)2010 Optical Society of America

OCIS codes: (140.3390) Laser materials processing; (260.7120) Ultrafast phenomena
\end{abstract}

The interaction of femtosecond laser pulses with transparent media has attracted considerable interest due to its unique properties. It was demonstrated that above a certain threshold self-assembled nano-grating in silica glass can be created [1]. Such subwavelength (for visible light) structure acts as a uniaxial crystal, where optical axes are aligned parallel and perpendicular to the grating corrugation. Several types of devices have been already demonstrated employing self-assembled nano-gratings: including birefringent Fresnel zone plate [2], nano-fluidic channels [3], rewritable optical memory and polarization selective waveguides [4]. Anisotropic properties were also exploited to create polarization selective binary computer-generated holograms [5]. Space-variant birefringence structures were fabricated using photolithographic methods [6]. Here we report first demonstration of femtosecond laser induced self-assembled nanostructuring for fabrication of space-variant birefringence structures embedded in silica glass, which could act as a beam splitters, polarization state converters or coronographs.

Regeneratively amplified, mode-locked Yb:KGW based femtosecond laser system (Pharos, Light Conversion Ltd.) operating at $1030 \mathrm{~nm}$ and delivering pulses of $270 \mathrm{fs}$ at $200 \mathrm{kHz}$ repetition rate was used in the experiments. The pulse energy was set to $0.5 \mu \mathrm{J}$, where nano-grating is known to be formed. The light beam was focused via a $\times 100$ (0.7 NA) Mitutoyo M Plan Apo NIR HR objective $70 \mu \mathrm{m}$ below the surface of a fused-silica plate. The sample was placed onto a computer controlled XYZ Aerotech translation stage. The control of the incident beam polarization azimuth was performed with achromatic half-wave plate mounted into motorized rotation stage. The grating was produced by subsequently writing $1 \mathrm{~mm}$ long lines at a $200 \mu \mathrm{m} / \mathrm{s}$ speed separated by $2 \mu \mathrm{m}$ (Fig. 1).

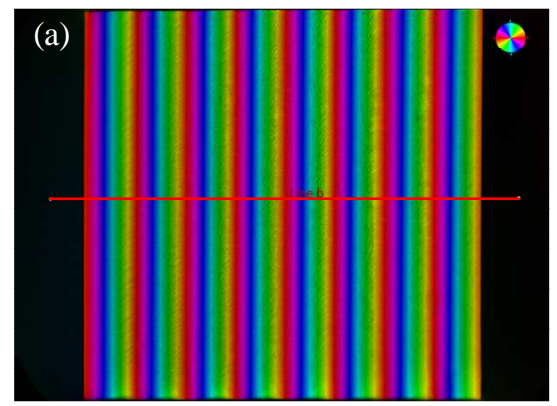

(b)

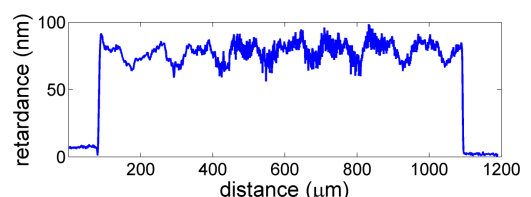

(c)

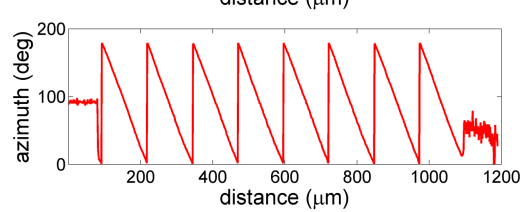

Fig. 1. (a) Quantitative birefringence microscopy image of polarization diffraction grating. Pseudo color indicates azimuth of the slow axis; (b) retardance and (c) azimuth profiles taken along the red line.

The induced retardance was around $90 \mathrm{~nm}$ (Fig. 1) at $515 \mathrm{~nm}$ wavelength. The desired variation of the slow axis was confirmed with quantitative birefringence microscope (Abrio, CRi Inc.). Diffraction properties were explored with Nd:YAG $(532 \mathrm{~nm})$ and $\mathrm{HeNe}(632.6 \mathrm{~nm}) \mathrm{CW}$ lasers. Images of far field diffraction were taken for different incident polarizations (Fig. 2). Different diffraction pattern with respect of the incident polarization state can be readily seen. The linear polarization was diffracted into three beams $(0,-1,1$ orders). For $632.6 \mathrm{~nm}$ the ratio of the amplitudes was 0.12:1:0.11. For left hand circular polarization only 0 and 1 orders were present (amplitude ratio 0.21:1:0), while right hand circular polarization resulted in 0 and -1 diffraction orders (amplitude ratio $0: 1: 0.18)$. This proved that the observed diffraction was caused by space variant polarization of the written structure. The average retardance value for $632.6 \mathrm{~nm}$ estimated on the basis of measured diffraction orders ratio was about $89 \mathrm{~nm}$, what is in good agreement with independent birefringence measurements. 
In our experimental conditions retardance over $200 \mathrm{~nm}$ can be achieved during single line scanning by extending focal distance via spherical aberrations or lower NA objective. Alternatively, higher values could be reached by writing multiple layers.

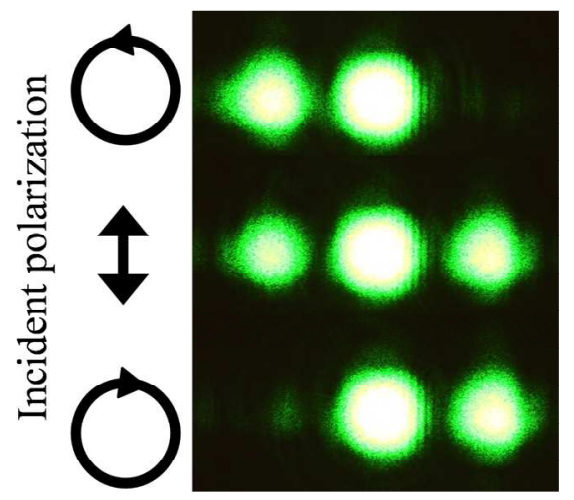

Fig. 2. Far-field diffraction images for linear and circular incident polarizations. Incident polarization state is indicated on the left side.

We also observed that if additional retardance is added to the polarization grating, the affected zone appears to be colored when visualizing in crossed polarizers (Fig. 3). This coloration is well known in polarization microscopy, which is present when optical path length difference due to birefringence is in the order of wavelength. Color images could be printed inside glass with resolution below one micron by controlling induced birefringence. This technique can be potentially applied for security marking or optical frequency filtering.
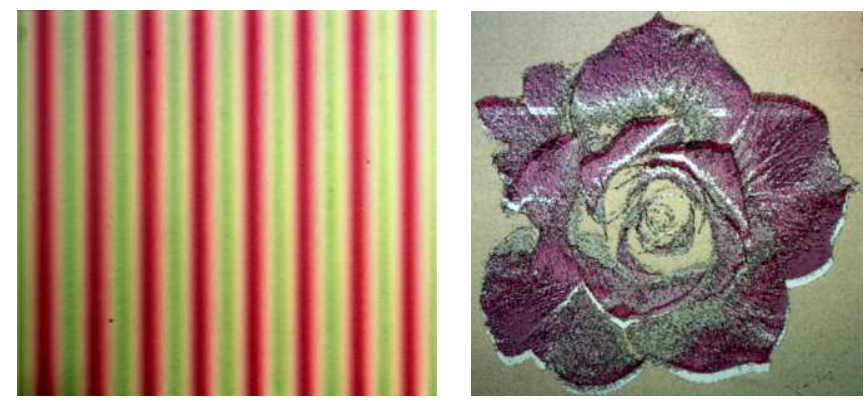

Fig. 3. (Left) Coloration of birefringent structure in crossed polarizers when additional constant retarder is added. (Right) Birefringent rose written in silica glass with femtosecond laser in $1 \mathrm{~mm} \times 1 \mathrm{~mm}$ square.

In summary, exploiting the ability of femtosecond lasers to create anisotropic modification inside silica glass, we fabricated polarization diffraction grating operating in visible light range. This approach provides advantages over current polarization diffractive optical elements fabrication techniques being single step process and enabling fabrication of structures with complex three dimensional geometries.

\section{References}

[1] Y. Shimotsuma, P. G. Kazansky, J. Qiu, and K. Hirao, "Self-organized nanogratings in glass irradiated by ultrashort light pulses," Phys. Rev. Lett. 24, 2474051-2474054 (2003).

[2] E. Bricchi, J. D. Mills, P. G. Kazansky, B. G. Klappauf, and J. J. Baumberg, "Birefringent Fresnel zone plates in silica fabricated by femtosecond laser machining," Opt. Lett. 27, 2200-2202 (2002).

[3] R. Taylor, C. Hnatovsky, and E. Simova, "Applications of femtosecond laser induced self-organized planar nanocracks inside fused silica glass," Laser Photon. Rev. 2, 26-46 (2008).

[4] G. Cheng, K. Mishchik, C. Mauclair, E. Audouard, and R. Stoian, "Ultrafast laser photoinscription of polarization sensitive devices in bulk silica glass," Opt. Express 17, 9515-9525 (2009).

[5] W. J. Cai, A. R. Libertun, and R. Piestun, "Polarization selective computer-generated holograms realized in glass by femtosecond laser induced nanogratings," Opt. Express 14, 3785-3791 (2006).

[6] E. Hasman, Z. Bomzon, A. Niv, G. Biener, and V. Kleiner, "Polarization beam-splitters and optical switches based on space-variant computer-generated subwavelength quasi-periodic structures," Opt. Commun. 209, 45-54 (2002). 